\title{
BMJ Open Protocol for an observational cohort study identifying factors predicting accurately end of life in dementia with Lewy bodies and promoting quality end-of-life experiences: the PACE- DLB study
}

Melissa J Armstrong (D) , ${ }^{1}$ Henry L Paulson, ${ }^{2}$ Susan M Maixner, ${ }^{3}$ Julie A Fields, ${ }^{4}$ Angela M Lunde, ${ }^{4}$ Bradley F Boeve, ${ }^{5}$ Carol Manning, ${ }^{6}$ James E Galvin, ${ }^{7}$ Angela S Taylor, ${ }^{8}$ Zhigang $\mathrm{Li}^{9}$

To cite: Armstrong MJ, Paulson HL, Maixner SM, et al. Protocol for an observational cohort study identifying factors predicting accurately end of life in dementia with Lewy bodies and promoting quality end-of-life experiences: the PACE-DLB study. BMJ Open 2021;11:e047554. doi:10.1136/ bmjopen-2020-047554

- Prepublication history for this paper is available online. To view these files, please visit the journal online (http://dx.doi. org/10.1136/bmjopen-2020047554).

Received 02 December 2020 Accepted 14 May 2021

D) Check for updates

(c) Author(s) (or their employer(s)) 2021. Re-use permitted under CC BY-NC. No commercial re-use. See rights and permissions. Published by BMJ.

For numbered affiliations see end of article.

Correspondence to Dr Melissa J Armstrong; Melissa.Armstrong@neurology. ufl.edu

\section{ABSTRACT}

Introduction Dementia with Lewy bodies (DLB) is one of the most common degenerative dementias. Despite the fact that most individuals with DLB die from complications of the disease, little is known regarding what factors predict impending end of life or are associated with a quality end of life.

Methods and analysis This is a multisite longitudinal cohort study. Participants are being recruited from five academic centres providing subspecialty DLB care and volunteers through the Lewy Body Dementia Association (not receiving specialty care). Dyads must be US residents, include individuals with a clinical diagnosis of DLB and at least moderate-to-severe dementia and include the primary caregiver, who must pass a brief cognitive screen. The first dyad was enrolled 25 February 2021; recruitment is ongoing. Dyads will attend study visits every 6 months through the end of life or 3 years. Study visits will occur inperson or virtually. Measures include demographics, DLB characteristics, caregiver considerations, quality of life and satisfaction with end-of-life experiences. For dyads where the individual with DLB dies, the caregiver will complete a final study visit 3 months after the death to assess grief, recovery and quality of the end-of-life experience. Terminal trend models will be employed to identify significant predictors of approaching end of life (death in the next 6 months). Similar models will assess caregiver factors (eg, grief, satisfaction with end-of-life experience) after the death of the individual with DLB. A qualitative descriptive analysis approach will evaluate interview transcripts regarding end-of-life experiences.

Ethics and dissemination This study was approved by the University of Florida institutional review board (IRB202001438) and is listed on clinicaltrials.gov (NCT04829656). Data sharing follows National Institutes of Health policies. Study results will be disseminated via traditional scientific strategies (conferences, publications) and through collaborating with the Lewy Body Dementia Association, National Institute on Aging and other partnerships.
Strengths and limitations of this study

- The Identifying Factors Predicting ACcurately Endof-Life in Dementia withLewy Bodies and Promoting Quality End-of-Life Experiences (PACE-DLB) study is a multisite longitudinal observational cohort study that will follow individuals with at least moderately advanced DLB and their caregivers for 3 years or until 3 months after the death of the person with DLB, providing important insight into patient and caregiver predictors of the final months of life in DLB.

- The PACE-DLB study will also investigate factors associated with a quality end of life in DLB and caregiver experiences after the death of the person with DLB, resulting in identification of opportunities for improving end-of-life care.

- The use of both virtual and in-person visits has strengths and limitations; this approach allows for recruitment of dyads who do or do not receive subspecialty care and recruitment of individuals with DLB too disabled to attend in-person study visits, but results in some differences in administration of study measures (though all measures will be verbally administered by study staff).

- The PACE-DLB study is US based, so issues relating to palliative and hospice care-or other aspects of healthcare-may not fully generalise to international locations.

\section{INTRODUCTION}

\section{Context and unmet needs}

Dementia with Lewy bodies (DLB) is one of the most common degenerative dementias after Alzheimer disease (AD) dementia. Despite DLB prevalence, evidence regarding prognosis and natural history is scarceparticularly when considering later stagesand represents a major unmet need. ${ }^{1}$ Known 
barriers to quality end-of-life care in dementia include lack of physician knowledge, prognostic uncertainty, difficulty assessing symptoms, and lack of physician recognition of terminal dementia stages and dementia as a cause of death. ${ }^{2}$ In DLB, fewer than half of caregivers reported discussions with a physician about what to expect at the end of life $(40 \%$ had discussions, only $22 \%$ to a helpful degree). ${ }^{3}$ Lack of knowledge regarding what to expect about the end of life in DLB-particularly the fact that DLB is terminal-was also a contributor to poor end-oflife experiences described in caregiver interviews. ${ }^{4}$

Improved end-of-life care and research are needed across dementias, ${ }^{5}$ but existing research focused largely on individuals with advanced dementia living in facilities and enrolled individuals with various dementias, particularly AD and mixed dementia ${ }^{6-9}$ Individuals with Lewy body dementia—an umbrella diagnosis including DLB and Parkinson disease dementiaaccounted for fewer than $3 \%$ of participants in these studies. ${ }^{7-9}$ Experiences for patients and family caregivers are likely to be different between $\mathrm{AD}$ and DLB. Individuals with DLB have shorter survival and die at a younger age than those with AD dementia. ${ }^{1011}$ Individuals with Lewy body dementia also have more than double the likelihood of respiratory death as those with AD dementia. ${ }^{12}$ Most individuals with DLB die of the dementia itself/failure to thrive $(72 \%)$ or pneumonia/ aspiration (23\%), which is commonly DLB related. ${ }^{3}$ Terminal dementia symptoms (eg, stopping speaking, inability to mobilise independently, requiring help with activities of daily living, dysphagia or stopping eating) likely overlap between dementias, but DLB-specific symptoms ${ }^{13}$ such as worsening hallucinations, parkinsonism, and daytime sleepiness were identified in caregiver interviews as additional endof-life features in DLB. ${ }^{4}$ These symptoms are missing from current tools predicting end of life in dementia (table 1). ${ }^{1415}$ Additionally, current tools have limitations even in dementia more generally. Medicare dementia hospice guidelines, which incorporate

Table 1 Predictors of end of life in dementia

\begin{tabular}{|c|c|c|c|}
\hline & $\begin{array}{l}\text { US medicare hospice benefit } \\
\text { Guidelines }^{15}\end{array}$ & ADEPT Score ${ }^{14}$ & $\begin{array}{l}\text { Interviews with DLB } \\
\text { caregivers }^{4}\end{array}$ \\
\hline Population included & Dementia & $\begin{array}{l}\text { Nursing home residents with } \\
\text { advanced dementia }\end{array}$ & DLB \\
\hline Increasing age & & $\checkmark$ & \\
\hline Male sex & & $\checkmark$ & \\
\hline Functional dependence & $\checkmark$ & $\checkmark$ & \\
\hline Unable to ambulate & $\checkmark$ & & $\checkmark$ \\
\hline Increased falls & & & $\checkmark$ \\
\hline Bedfast most of day & & $\checkmark$ & $\checkmark$ \\
\hline Generalised weakness & & & $\checkmark$ \\
\hline Increased rigidity & & & $\checkmark$ \\
\hline Limited speech & $\checkmark$ & & $\checkmark$ \\
\hline Increased hallucinations & & & $\checkmark$ \\
\hline Worsened daytime somnolence & & & $\checkmark$ \\
\hline Incontinence & $\checkmark$ & $\checkmark$ & 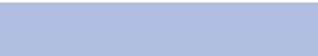 \\
\hline Shortness of breath & & $\checkmark$ & \\
\hline Insufficient oral intake & $\checkmark$ & $\checkmark$ & $\checkmark$ \\
\hline Weight loss/low BMI & & $\checkmark$ & $\checkmark$ \\
\hline Hospitalisation or procedure & & & $\checkmark$ \\
\hline Pressure ulcers & $\checkmark$ & $\checkmark$ & \\
\hline $\begin{array}{l}\text { Swallowing difficulties, } \\
\text { aspiration pneumonia }\end{array}$ & $\checkmark$ & & $\checkmark$ \\
\hline $\begin{array}{l}\text { Pyelonephritis, upper urinary } \\
\text { tract infection }\end{array}$ & $\checkmark$ & & \\
\hline Sepsis & $\checkmark$ & & \\
\hline Congestive heart failure & & $\checkmark$ & \\
\hline
\end{tabular}

ADEPT, Advanced Dementia Prognostic Tool; BMI, body mass index; DLB, dementia with Lewy bodies. 
Functional Assessment Staging (FAST), had a sensitivity of only 0.20 for 6 -month mortality when applied to a nursing home population with advanced dementia (area under the curve (AUC) 0.55; specificity 0.89). ${ }^{14}$ The Advanced Dementia Prognostic Tool (ADEPT), developed in a nursing home setting, performed only marginally better (AUC 0.58; sensitivity 0.27; specificity 0.89) ${ }^{14}$ These knowledge gaps limit the ability of individuals with DLB and their families to anticipate and adequately plan for care transitions and needs.

\section{Study aims}

The current study aims to investigate end-of-life experiences for patient/caregiver dyads living with DLB with or without subspecialty care. The study aims to determine patient and caregiver predictors of end of life in DLB (defined as death within 6 months, based on the window for US Medicare coverage for hospice services). Additionally, the study will identify the impact of demographics, healthcare factors, DLB symptoms and caregiver factors on patient quality of life (QoL) and caregiver experiences at the end of life of the person living with DLB.

The primary outcome for aims 1 and 2 is death within 6 months. We hypothesise that predictors of death within 6 months for individuals with DLB will include symptoms (eg, worsening hallucinations, fluctuations) not captured in existing dementia end-of-life prediction tools. We also hypothesise that there will be worsening caregiver burden, depression, grief and QoL preceding the last 6 months of life, controlling for variables such as resilience, support, and living situation. Finally, we hypothesise that location (rural, urban, suburban), provider experience (eg, specialist vs not), patient residence (eg, home, skilled nursing), degree of behavioural symptoms, caregiver type and caregiver characteristics (self-efficacy, perceived social support, resilience and coping) will affect patient and caregiver experiences at the patient's end of life, including patient and caregiver QoL and caregiver burden (primary outcome for predeath analysis), wellbeing, depression, grief and satisfaction with end-oflife care (primary outcome for postdeath analysis).

\section{METHODS AND ANALYSIS \\ Study design}

Identifying Factors Predicting ACcurately End-of-Life in Dementia withLewy Bodies and Promoting Quality End-of-Life Experiences (PACE-DLB) is an observational longitudinal cohort study involving five Lewy Body Dementia Association Research (LBDA) Research Centers of Excellence in the USA (University of Florida, University of Michigan, Mayo Clinic Rochester, University of Virginia, University of Miami) and recruitment of a virtual cohort (not receiving subspecialty care) through the LBDA. There are two cohort types: (1) cohorts with or without subspecialty care and (2) division of the overall cohort into a group where the individual with DLB lives 6 months after the study visit vs the group where the individual with DLB dies during the 6 months between study visits (figure 1). The cohort where the individual with DLB lives with continued disease progression versus the end-of-life cohort will change throughout the study based on the timing of death. The statistical approaches account for this. Study visits occur every 6 months and are completed either in-person or virtually (via phone or videoconferencing). After the individual with DLB dies, the caregiver will complete a virtual end-of-study visit 3 months after the patient's death to assess grief and recovery. A 3-month postdeath follow-up was chosen based on research showing that caregiver depression declines significantly in the 13 weeks after the death of an individual with dementia ${ }^{16}$ and bereaved family member survey responses were stable between 3 and 9 months after

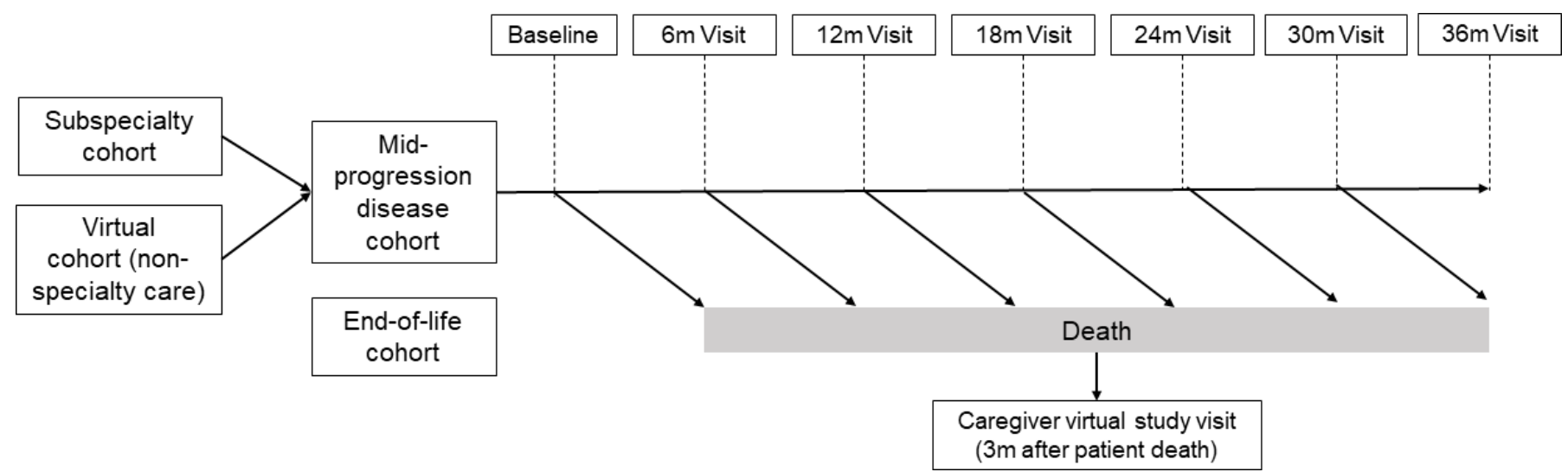

Figure 1 Identifying Factors Predicting ACcurately End-of-Life in Dementia withLewy Bodies and Promoting Quality End-ofLife Experiences (PACE-DLB) study design: the PACE-DLB study will recruit 75 dyads (individuals with DLB and their primary informal caregiver) from subspecialty clinics and 75 dyads not receiving subspecialty care. After meeting inclusion criteria, dyads will have a baseline study visit and then return for follow-up study visits every 6 months for 3 years or until the death of the individual with DLB. Caregivers of individuals with DLB who die during the study will complete a final study visit 3 months after the death of the person with DLB. DLB, dementia with Lewy bodies. 
the death of individuals on hospice. ${ }^{17}$ The first dyad was enrolled 25 February 2021 and recruitment is ongoing.

\section{Study participants}

Inclusion criteria include: (1) patient and caregiver willing to participate as a dyad, (2) US residents, (3) patient with a clinical diagnosis of DLB, (4) patient with at least moderate severity dementia as assessed by the Quick Dementia Rating System (QDRS, score of $>12$ suggestive of moderate dementia), ${ }^{18}(5)$ patient expected to live at least 6 months and (6) caregiver Telephone Interview for Cognitive Status score of $>31$ to ensure that the caregiver is able to reliably complete study visits. ${ }^{19}$ Diagnosis of DLB will be supported using the Lewy Body Composite Risk Score (LBCRS, score $\geq 3$ consistent with DLB) ${ }^{20}$

The participating caregiver must be the person providing the majority of the patient's informal care (whether the individual with DLB is living at home or in a facility) and attending the majority of the patient's clinical visits. The caregiver can represent any relationship with the individual with DLB (eg, spouse, child). The type of relationship will be captured to assess the impact of this relationship/role. The participating caregiver must remain the same throughout the study. If the primary caregiver changes (eg, due to caregiver death), that dyad will drop out of the study. The location of the person with DLB (eg, home, facility) will be tracked for its influence on outcomes and its relationship with informal caregiver responsibilities.

By including individuals with DLB with at least moderate dementia severity, it is anticipated that a majority of participants will reach end of life over the 3 years of the study. Median duration from diagnosis to death in DLB is 3-4 years. ${ }^{311} 21$ Thus, even if enrolling individuals early in their disease course, half would likely approach end of life during a 3-year study. By focusing on individuals already in at least moderate dementia stages, the proportion of individuals progressing to end of life during the study will be higher. The study does not require that all participants progress to death during the 3-year period.

\section{Recruitment}

Study sites will recruit from patient-caregiver dyads presenting to their LBDA Research Centre of Excellence clinics (subspecialty cohort). For the virtual cohort without subspecialty care, participants will be recruited through the LBDA website, emails to individuals subscribed to the LBDA's mailing list and social media posts. The study is also listed on clinicaltrials.gov (NCT04829656).

\section{Study measures}

We prioritised measures used by existing DLB cohorts in the USA (DLB Consortium, National Alzheimer's Coordinating Center (NACC) cohorts) so that data can be combined with other cohorts to answer future research questions. For PACE-DLB, measures are identical for in-person and virtual visits except that the Unified Parkinson Disease Rating Scale (UPDRS) motor subscale ${ }^{22}$ will only be completed at in-person visits. All measures are caregiver-completed except the motor UPDRS and QoL$\mathrm{AD}$, which is completed by both the patient (if able) and the caregiver.

Demographics include patient and caregiver gender, sex, age, racial/ethnic background, education, disease duration, hours spent caregiving and the relationship of the caregiver to the patient. Collected background will also include details regarding the medical team caring for the person with DLB, such as clinician types (eg, primary care physician, geriatrician, general/specialty neurologist) and ancillary services used (eg, therapy, social work/care coordination, palliative care). Transitions will be noted, including home to hospital, home to hospice, home to nursing home, etc. ${ }^{23}$

Formal scales assess patient and caregiver experiences (table 2). The LBCRS will measure the breadth of DLB symptoms present. $^{20}$ While designed as a screening instrument rather than a measure of disease severity, this interview-based scale will provide a way to assess disease scope using an instrument that can be completed verbally. Cognitive function will be assessed via the QDRS, which can be converted to a CDR Dementia Staging Instrument score but takes substantially less time $(\sim 3-5 \mathrm{~min})$ to complete. ${ }^{18}$ The Neuropsychiatric Inventory Questionnaire ${ }^{2425}$ is the most commonly used measure for assessing behavioural and psychological symptoms of dementia in people with DLB. The Mayo Fluctuations $\mathrm{Scale}^{26}$ and Clinician Assessment of Fluctuations ${ }^{27}$ will measure fluctuations. The Mayo Sleep Questionnaire ${ }^{28}$ and Epworth Sleepiness Scale ${ }^{29}$ will assess aspects of sleep relevant to DLB (eg, symptoms consistent with REM sleep behaviour disorder, daytime sleepiness). The Autonomic Systems Checklist from the NACC Lewy body dementia module will identify the presence of autonomic symptoms. QoL of the individual with DLB will be assessed using participant and caregiver-completed versions of the QoL-AD scale, validated for individuals with dementia. ${ }^{30}$ This scale is not AD-specific and was used previously with individuals with DLB. $^{31}$

To investigate clinical factors potentially contributing to quality end-of-life care in dementia, we will assess medication prescribing by collecting concomitant medications and applying the Anticholinergic Cognitive Burden scale $^{3233}$ and STOPP criteria for potentially inappropriate prescribing. ${ }^{34}$ Caregiver-reported medication use will help inform whether use of certain 'hospice pack' medications, such as haloperidol or lorazepam, is associated with different end-of-life experiences. To assess estimates of approaching end of life, we will use the FAST scale used in Medicare dementia hospice guidelines ${ }^{15}$ and the ADEPT score. ${ }^{14}$

Caregiver QoL will be measured using the Carer Wellbeing and Support Questionnaire, recently identified as the most appropriate QoL instrument for informal caregivers for individuals with dementia. ${ }^{35}$ Caregiver depression will be measured with the Centre for Epidemiologic Studies Depression Scale, also used in prior end-of-life 
Table 2 Measures for the PACE-DLB study

\begin{tabular}{|c|c|}
\hline Category & Measure \\
\hline DLB symptoms & Lewy Body Composite Risk Score \\
\hline Global cognitive rating & Quick Dementia Rating System \\
\hline Motor function & Unified Parkinson's Disease Rating Scale Part III (motor subscale)* \\
\hline Neuropsychiatric & Neuropsychiatric Inventory-Questionnaire* \\
\hline Fluctuations & $\begin{array}{l}\text { Mayo Fluctuations Scale* } \\
\text { Clinician Assessment of Fluctuations }\end{array}$ \\
\hline Sleep (including RSBD, daytime sleepiness) & $\begin{array}{l}\text { Mayo Sleep Questionnaire (caregiver-reported)* } \\
\text { Epworth Sleepiness Scale }\end{array}$ \\
\hline Autonomic function & Autonomic symptoms checklist* \\
\hline Appropriate drug prescribing & $\begin{array}{l}\text { Concomitant medications } \\
\text { Anticholinergic Cognitive Burden scale } \\
\text { STOPP criteria }\end{array}$ \\
\hline Measures of approaching end-of-life & $\begin{array}{l}\text { Functional Assessment Staging } \\
\text { US Medicare dementia hospice guidelines } \\
\text { Advanced Dementia Prognostic Tool Score }\end{array}$ \\
\hline Patient quality of life & Quality of Life in Alzheimer's disease (individual with DLB, caregiver) \\
\hline Caregiver quality of life & Carer Well-Being and Support Questionnaire \\
\hline Caregiver depression & Centre for Epidemiologic Studies Depression Scale \\
\hline Caregiver self-efficacy & Revised Scale for Caregiving Self-Efficacy \\
\hline Caregiver resilience & The Resilience Scale \\
\hline Caregiver social support & Multidimensional Scale of Perceived Social Support \\
\hline Caregiver coping & Brief Coping Orientation to Problems Experienced \\
\hline Caregiver burden & Short Zarit Revised Burden Interview \\
\hline Caregiver anticipatory grief & Meuser-Marwit Caregiver Grief Inventory-Short Form \\
\hline Caregiver bereavement & Texas Revised Inventory of Grief \\
\hline End-of-life care experiences & National Health and Ageing Trends Study last month of life survey \\
\hline
\end{tabular}

*Used by DLB Consortium and/or National Alzheimer's Coordinating Center (Unified Data Set or Lewy body dementia module V.3).

DLB, dementia with Lewy bodies; LBCRS, Lewy Body Composite Risk Score.

dementia bereavement research. ${ }^{16} 36$ The Revised Scale for Caregiving Self-Efficacy covers topics relating to confidence obtaining respite, responding to disruptive behaviours, and controlling upsetting thoughts about caregiving. ${ }^{37} 38$ The Multidimensional Scale of Perceived Social Support is a social support scale ${ }^{3940}$ recommended by the National Alzheimer's Disease Resource Center. ${ }^{41}$ Coping strategies will be measured by the Brief Coping Orientation to Problems Experienced, ${ }^{42}$ also recommended by the National Alzheimer's Disease Resource Center $^{41}$ and used in prior studies with caregivers of individuals with dementia at the end of life. ${ }^{43}$ Caregivers will complete the shortened Zarit Revised Burden interview (sZBI),${ }^{44}$ used in prior studies with caregivers of individuals with DLB. ${ }^{45}$ Caregiver anticipatory grief will be measured with the Meuser-Marwit Caregiver Grief Inventory-Short Form, specifically developed for caregivers of individuals with dementia. ${ }^{46}$ Postdeath grief (bereavement) will be measured using the Texas Revised Inventory of Grief, used previously to measure postdeath grief of informal caregivers of individuals with dementia. ${ }^{36}$ Caregiver resilience will be measured using the Resilience Scale, the original resilience measure and one used previously in dementia caregiver studies. ${ }^{47} 48$ At the 3-month postdeath visit, caregivers will receive the National Health and Aging Trends Study last month of life interview, ${ }^{49}$ which queries endof-life experiences relating to pain, dyspnoea, anxiety/ sadness, decision making, needs, respect and communication, and which solicits an overall rating of end-of-life care. As with a prior study, ${ }^{50}$ we will supplement the last month of life questionnaire-based interview with semistructured interview questions to further investigate endof-life experiences.

\section{Study visits}

Measures will be administered verbally to minimise differences in conduct between the virtual and in-person groups. Verbal administration will be used across measures because some older caregivers would be uncomfortable with self-administered electronic form completion. Visits are expected to last 2-3 hours. Multiple approaches will be used to limit survey fatigue and burden. For in-person visits, breaks and snacks will be provided to limit fatigue. For virtual participants who may have difficulty being 
on the phone or videoconference for 2-3 hours continuously, we will encourage breaks and if needed, provide the option of completing the scales over two sessions, one focusing on the patient experience and one focusing on the caregiver experience.

Participants will complete study visits every 6 months either in person or virtually for the 3 years of the study or until the death of the person with DLB. If the person with DLB dies, the caregiver will complete one final study visit 3 months after the death of the individual with DLB (figure 1). The postdeath study visit will include the Center for Epidemiologic Studies Depression Scale, Resilience Scale, Texas Revised Inventory of Grief, National Health and Aging Trends Study last month of life interview (questionnaire based), and a semistructured interview about end-of-life experiences.

\section{Data collection}

Coordinators will enter data directly into electronic case report forms. Study data will be collected and managed using REDCap (Research Electronic Data Capture) tools hosted at the University of Florida. ${ }^{51}{ }^{52}$ REDCap is a secure, web-based software platform designed to support data capture for research studies, providing (1) an intuitive interface for validated data capture; (2) audit trails for tracking data manipulation and export procedures; (3) automated export procedures for seamless data downloads to common statistical packages and (4) procedures for data integration and interoperability with external sources. The postdeath caregiver interviews will be audiorecorded and professionally transcribed.

\section{Analysis}

\section{Sample size}

Target sample size for the overall cohort was based on a pairwise comparison of the sZBI score before and after entering the last 6 months of life. If recruiting 128 dyads and using an sZBI SD reported previously in DLB (8.8) and assuming the within-participant correlation of the outcome ranges from 0.2 to 0.8 , the minimum detectable pairwise difference ranges from 1.4 to 2.7 with $80 \%$ power and two-sided type I error rate of 0.05 . This standardised minimum detectable difference range is from $0.16 \mathrm{SD}$ to $0.31 \mathrm{SD}$ which gives sufficient power for the study. To account for a 15\% drop-out, 150 total dyads will be enrolled. Target enrollment will be split evenly between in-person and virtual cohorts, with a target of 75 dyads recruited from the five LBDA Research Center of Excellence sites and 75 virtual dyads recruited through the LBDA.

\section{Overall analysis}

Descriptive statistical measures including mean, median, $\mathrm{SD}$, minimum and maximum values of continuous variables and frequency of categorical variables will be generated. Appropriate transformations (eg, natural-log) will be applied as needed to comply with normality. Data visualisation such as scatter plots will be implemented.

\section{Analysis of predictors of end of life}

A variable that predicts the end of life will be defined as a variable changing substantially (eg, quick drop of QoL) once the patient enters last 6 months of life. Statistical models will estimate the longitudinal trajectories of potential candidate variables to identify those with significantly different trajectory patterns in the last 6 months of life. A preliminary analysis will evaluate candidate variables in the decedents subsample and compare scores before and after entering the last 6 months of life to identify variables that have substantial changes. Subsequently, a terminal trend model (TTM $)^{53} 54$ will estimate the terminal trends of the candidate variables to test the difference of longitudinal pattern before and after entering last 6 months of life to select the variables that are significant predictors of end of life. TTM can include participants who drop out or do not die. By modelling the longitudinal and survival data simultaneously, TTM addresses the cohort effect of analysing a subsample (eg, decedents only) of the entire study because every patient contributes to the joint analysis. TTM can also alleviate the burden of missing data due to censoring. A dynamic prediction model will identify variables that might predict conditional survival probability, which could be helpful for identifying variables predicting the last 6 months of life. All these analyses will be adjusted for potential confounders such as resilience, support, living situation and DLB severity. The cohorts will be combined for the analyses but we will also conduct the analyses in cohorts with and without subspecialty care to check cohort-specific effects. Stratified analyses will examine potential differences relating to sex or gender.

\section{Analysis of factors associated with quality end-of-life experiences}

The outcome measures assessing end-of-life experiences include the QoL of the person with DLB (at the last predeath visit) and caregiver-reported measures obtained at the last predeath visit and the 3 months postdeath visit including caregiver burden, well-being and support, depression, grief and satisfaction (as captured by the last month of life survey). Independent variables of interest include patient demographics (eg, age, gender, sex, race/ ethnicity, disease duration, level of education), caregiver demographics (eg, age, gender, sex, race/ethnicity, relationship to patient, level of education), disease characteristics (eg, overall symptom burden, cognition, motor function, neuropsychiatric symptoms, functional status, fluctuations, sleep-related symptoms, autonomic symptoms, medications), elements of care (rural/urban, type of healthcare provider, patient residence) and caregiver measures (self-efficacy, social support, coping skills). Associations/correlations of independent variables and the dependent variables/outcomes will be assessed using nonparametric methods such as Wilcoxon rank sum test for binary demographic variables, Kruskal-Wallis test for categorical demographic variables and Spearman correlation for continuous demographic variables. 
Non-parametric scatter plots with splines will be used to visualise possibly non-linear relationships.

For multivariate analyses, TTM will test the (possibly time-varying) effects of demographic variables on the longitudinal trajectories of end-of-life measures. Demographic variables will be modelled as independent variables simultaneously in the TTM approach to control for potential confounding effects between the demographics and the end-of-life measures will be dependent outcomes. Demographics that have significant effects on the trajectories of end-of-life outcomes will be selected based on effect size and significance level (indicated by p values). When necessary (eg, many demographic variables), regularisation approaches including least absolute shrinkage and selection operator (LASSO), ${ }^{55}$ minimax concave penalty (MCP $)^{56}$ and knockoff filter ${ }^{57}$ will be incorporated into the TTM approach to select independent variables that have larger impact on the outcomes. False discovery rate will be controlled by selecting tuning parameters with permutation approaches. Cross-validation such as 10 -fold cross-validation will be used in the regularisation approaches to validate the model by randomly splitting the data into partitions where the model is trained and tested in difference partitions. Unsupervised learning approaches $^{58}$ will also be used to identify clusters that might be associated with specific outcomes. Latent class models ${ }^{59}$ will be applied to identify latent clusters that might be associated with the trajectory of the outcomes. Potential confounders will be adjusted in all the analyses. Subsample analyses will assess for cohort and sex/gender effects.

Investigators will use a qualitative descriptive approach to analyse transcripts of the post-death caregiver interviews. The qualitative descriptive approach reports experiences without intending to generate or use theory. ${ }^{60}$

\section{Patient and public involvement}

The research questions for this study were informed by a survey and interviews with individuals whose loved ones died of DLB. Caregivers indicated that they did not know what to expect at the end of life in DLB and that this negatively impacted end-of-life experiences. ${ }^{34}$ Staff associated with the LBDA—a US-based advocacy organisation for individuals with DLB and their families-participated in study design and grant development. There was no community advisory board.

\section{Data monitoring}

Due to the minimal risk nature of this observational study, there is no external data and safety monitoring board. The principal investigator, coinvestigators and study staff will monitor study conduct internally. Consistency across sites will be achieved through study protocol training, regular study teleconferences with representatives from all sites, and site evaluations by the coordinating site. Each site will maintain participant screening and enrolment logs with contact information and other identifiable data. Data entered into the electronic case report forms will be deidentified apart from visit and death dates (required for the time-based analyses). Reports detailing study progress will be submitted to the National Institutes on Aging annually.

\section{Ethics and dissemination}

\section{Ethics approval}

This study is managed by a single Institutional Review Board at the University of Florida (IRB202001438) consistent with requirements from the National Institutes of Health.

\section{Informed consent}

Caregivers will consent for their own participation. Legally authorised representatives (who may or may not be the caregiver) will provide consent on behalf of the person with dementia. Each site will follow local (state) laws for determining the legally authorised representative. Individuals with DLB will be asked to provide assent if able. If a potential participant resists participating, he or she will not be enrolled. Informed consent will be obtained in manners following good clinical practice.

\section{Data sharing}

Consistent with data sharing regulations from the National Institutes of Health, investigators will make the final data and associated documentation available to users under a data-sharing agreement that provides for a commitment to: (1) use the data for research purposes only; (2) not identify any individual participant (the final dataset will be deidentified prior to release, but there remains the possibility of deductive disclosure of participants with specific characteristics); (3) use appropriate data security and (4) destroy the data after analyses are completed. PACE-DLB measures were chosen to facilitate combining DLB data sets to answer future research questions.

\section{Dissemination}

The results of this study are important to disseminate to individuals with DLB, caregivers, advocacy organisations and healthcare professionals. Traditional strategies for scientific dissemination will be utilised, including conference presentations and peer-reviewed publications. Results relevant for individuals living with DLB and caregivers will be disseminated through the Lewy Body Dementia Association, already a study partner, and through the National Institutes on Aging, which has educational webpages dedicated to end of life in DLB. The LBDA has existing strategies for communicating with populations living with DLB including webpages highlighting research results, webinars and social media use. Results will also be shared with the general population through mechanisms like the website 'The Conversation,' which partners with academic organisations to educate the public. 


\section{DISCUSSION}

This protocol outlines the methodology for the PACE-DLB study, a multicentre observational cohort study investigating patient-related and caregiver-related predictors of end of life in DLB and factors associated with a quality end of life. The study makes use of a classic longitudinal cohort model, but focuses on collecting data pertinent to the end of life rather than biomarker and natural history data, the focus of most longitudinal DLB cohorts. To overcome the limitation in many natural history studies in which participants are only enrolled at specialty centres (with negative implications for generalisability) and where consent to autopsy is sometimes required (further biasing enrolment), PACE-DLB uses a subspecialty care cohort and a virtual cohort without DLB subspecialty care to obtain a broad representation of experiences. In addition to improving generalisability, this approach allows an assessment of whether certain care settings (eg, primary care vs general neurology vs subspecialty care) impact end-of-life experiences. Designing visits to be compatible with phone-based or teleconference-based administration also facilitates following participants in the subspecialty cohort even if they are unable to come to clinic. Enrolling participants in dyads enables assessment of patientrelated outcomes but also caregiver outcomes relating to burden, resilience, and grief, before and after the death of the individual with DLB. Through this longitudinal study following dyads through the end-of-life period, the death of the person with DLB, and initial bereavement for the caregiver, PACE-DLB will identify predictors and experiences of end of life in DLB with various care experiences. This will improve prognostic and end-of-life counselling for individuals and families living with DLB and also identify areas that can be targeted to improve end-oflife experiences.

\author{
Author affiliations \\ ${ }^{1}$ Neurology, University of Florida College of Medicine, Gainesville, Florida, USA \\ ${ }^{2}$ Neurology, University of Michigan, Ann Arbor, Michigan, USA \\ ${ }^{3}$ Psychiatry, University of Michigan, Ann Arbor, Michigan, USA \\ ${ }^{4}$ Psychiatry and Psychology, Mayo Clinic Rochester, Rochester, Minnesota, USA \\ ${ }^{5}$ Neurology, Mayo Clinic Rochester, Rochester, Minnesota, USA \\ ${ }^{6}$ Neurology, University of Virginia, Charlottesville, Virginia, USA \\ ${ }^{7}$ Neurology, University of Miami Miller School of Medicine, Miami, Florida, USA \\ ${ }^{8}$ Lewy Body Dementia Association, Lilburn, Georgia, USA \\ ${ }^{9}$ Biostatistics, University of Florida College of Medicine, Gainesville, Florida, USA
}

Contributors MJA conceived and designed the study, serves as the principal investigator for the grant, drafted the protocol, and serves as the guarantor of the work. HLP, SMM, JAF, AML, BFB and CM provided feedback on study design, revised the protocol critically for important intellectual conduct, and serve as coinvestigators on the grant. JEG and AT provided feedback on study design, revised the protocol critically for important intellectual conduct and serve as consultants on the grant. ZL provided feedback on study design, designed the statistical analysis, revised the protocol critically for important intellectual conduct and serves as a coinvestigator on the grant. All authors provided approval for the protocol to be published.

Funding This work was supported by the National Institutes on Aging grant number R01AG068128.

Disclaimer The content is solely the responsibility of the authors and does not necessarily represent the official views of the National Institutes of Health/National Institutes on Aging.
Competing interests MJA receives research support from the NIA (P30AG047266, R01AG068128) and the Florida Department of Health (grant 20A08). She is the local PI of a Lewy Body Dementia Association Research Center of Excellence. She serves on the Scientific Advisory Board of the Lewy Body Dementia Association. HLP receives funding from the NIA (1P30AG053760) and is the local PI of a Lewy Body Dementia Association Research Center of Excellence. SMM has no competing interests. JAF receives research support from the NIA (U01NS100620, R01AG068128, R43AG65088). AML receives research support from the NIA (P30AG62677, R43AG65088). She is a Program Coordinator for the local a Lewy Body Dementia Association Coordinating Center Research Center of Excellence. BFB has served as an investigator for clinical trials sponsored by Biogen, Alector and EIP Pharma. He serves on the Scientific Advisory Board of the Lewy Body Dementia Association, Association for Frontotemporal Degeneration and Tau Consortium. He is the site PI of a Lewy Body Dementia Association Research Center of Excellence program, as well as coordinating center PI of the program. He receives research support from the $\mathrm{NIH}$, the Mayo Clinic Dorothy and Harry T. Mangurian Jr. Lewy Body Dementia Programme, and the Little Family Foundation. CM receives research support from ACL/DHHS (90ALGG0014-01-00), NIA/NIH (2SB1AG037357-04A1, R01-AG-054435), HRSA (U1QHP287440400) and DoD (AZ190036). She is the local PI of a Lewy Body Dementia Association Research Center of Excellence. JEG is the creator of the QDRS and the LBCRS. He is supported by grants from the National Institutes of Health (R01 AG069765, R01 AG057681, R01 NS101483, P30 AG059295, U54 AG06354, R01 AG056531, U01 NS100610, R01 AG056610, R01 AG054425, R01 AG068128) and the Leo and Anne Albert Charitable Trust. He is the local PI of the Lewy Body Dementia Association Research Center of Excellence at the University of Miami and serves on the Scientific Advisory Board of the Lewy Body Dementia Association. AT is an employee of the Lewy Body Dementia Association. ZL has no competing interests.

Patient and public involvement Patients and/or the public were involved in the design, or conduct, or reporting, or dissemination plans of this research. Refer to the Methods section for further details.

Patient consent for publication Not required.

Provenance and peer review Not commissioned; externally peer reviewed.

Open access This is an open access article distributed in accordance with the Creative Commons Attribution Non Commercial (CC BY-NC 4.0) license, which permits others to distribute, remix, adapt, build upon this work non-commercially, and license their derivative works on different terms, provided the original work is properly cited, appropriate credit is given, any changes made indicated, and the use is non-commercial. See: http://creativecommons.org/licenses/by-nc/4.0/.

ORCID iD

Melissa J Armstrong http://orcid.org/0000-0002-2163-1907

\section{REFERENCES}

1 Mueller C, Ballard C, Corbett A, et al. The prognosis of dementia with Lewy bodies. Lancet Neurol 2017;16:390-8.

2 Erel M, Marcus E-L, Dekeyser-Ganz F. Barriers to palliative care for advanced dementia: a scoping review. Ann Palliat Med 2017;6:365-79.

3 Armstrong MJ, Alliance S, Corsentino P, et al. Cause of death and end-of-life experiences in individuals with dementia with Lewy bodies. J Am Geriatr Soc 2019;67:67-73.

4 Armstrong MJ, Alliance S, Taylor A, et al. End-Of-Life experiences in dementia with Lewy bodies: qualitative interviews with former caregivers. PLoS One 2019;14:e0217039.

5 Perrar KM, Schmidt H, Eisenmann Y, et al. Needs of people with severe dementia at the end-of-life: a systematic review. J Alzheimers Dis 2015;43:397-413.

6 Mitchell SL, Kiely DK, Jones RN, et al. Advanced dementia research in the nursing home: the cascade study. Alzheimer Dis Assoc Disord 2006;20:166-75.

7 Toscani F, van der Steen JT, Finetti S, et al. Critical decisions for older people with advanced dementia: a prospective study in long-term institutions and district home care. J Am Med Dir Assoc 2015;16:535. e13-535.e20.

8 van der Steen JT, Ribbe MW, Deliens L, et al. Retrospective and prospective data collection compared in the Dutch end of life in dementia (DEOLD) study. Alzheimer Dis Assoc Disord 2014;28:88-94.

9 Sampson EL, Candy B, Davis S, et al. Living and dying with advanced dementia: a prospective cohort study of symptoms, service use and care at the end of life. Palliat Med 2018;32:668-81. 
10 Williams MM, Xiong C, Morris JC, et al. Survival and mortality differences between dementia with Lewy bodies vs Alzheimer disease. Neurology 2006;67:1935-41.

11 Price A, Faroog R, Yuan J-M, et al. Mortality in dementia with Lewy bodies compared with Alzheimer's dementia: a retrospective naturalistic cohort study. BMJ Open 2017;7:e017504.

12 Garcia-Ptacek S, Kåreholt I, Cermakova P, et al. Causes of death according to death certificates in individuals with dementia: a cohort from the Swedish dementia registry. J Am Geriatr Soc 2016;64:e137-42.

13 McKeith IG, Boeve BF, Dickson DW, et al. Diagnosis and management of dementia with Lewy bodies: fourth consensus report of the DLB Consortium. Neurology 2017;89:88-100.

14 Mitchell SL, Miller SC, Teno JM, et al. Prediction of 6-month survival of nursing home residents with advanced dementia using ADEPT vs hospice eligibility guidelines. JAMA 2010;304:1929-35.

15 Mitchell SL. Advanced dementia. N Engl J Med 2015;373:2533-40.

16 Schulz R, Mendelsohn AB, Haley WE, et al. End-Of-Life care and the effects of bereavement on family caregivers of persons with dementia. N Engl J Med 2003;349:1936-42.

17 DiBiasio EL, Clark MA, Gozalo PL, et al. Timing of survey administration after hospice patient death: stability of bereaved respondents. J Pain Symptom Manage 2015;50:17-27.

18 Galvin JE. The quick dementia rating system (QDRS): a rapid dementia staging tool. Alzheimers Dement 2015;1:249-59.

19 Lacruz M, Emeny R, Bickel H, et al. Feasibility, internal consistency and covariates of TICS-m (telephone interview for cognitive statusmodified) in a population-based sample: findings from the KORAAge study. Int J Geriatr Psychiatry 2013;28:971-8.

20 Galvin JE. Improving the clinical detection of Lewy body dementia with the Lewy body composite risk score. Alzheimers Dement 2015;1:316-24.

21 Walker Z, Allen RL, Shergill S, et al. Three years survival in patients with a clinical diagnosis of dementia with Lewy bodies. Int J Geriatr Psychiatry 2000;15:267-73.

22 Fahn S, Elton RL, UPDRS program members. Unified Parkinson's disease rating scale. In: Fahn S, Marsden CD, Goldstein M, et al, eds. Recent developments in Parkinson's disease. Vol. 2. Florham Park, NJ: Macmillan Healthcare Information, 1987: 153-63.

23 Makaroun LK, Teno JM, Freedman VA, et al. Late transitions and bereaved family member perceptions of quality of end-of-life care. $J$ Am Geriatr Soc 2018;66:1730-6.

24 Cummings JL, Mega M, Gray K, et al. The neuropsychiatric inventory: comprehensive assessment of psychopathology in dementia. Neurology 1994;44:2308-14.

25 Kaufer DI, Cummings JL, Ketchel P, et al. Validation of the NPI-Q, a brief clinical form of the neuropsychiatric inventory. $J$ Neuropsychiatry Clin Neurosci 2000;12:233-9.

26 Ferman TJ, Smith GE, Boeve BF, et al. Dlb fluctuations: specific features that reliably differentiate DLB from AD and normal aging. Neurology 2004;62:181-7

27 Walker MP, Ayre GA, Cummings JL, et al. The clinician assessment of fluctuation and the one day fluctuation assessment scale. two methods to assess fluctuating confusion in dementia. Br J Psychiatry 2000;177:252-6.

28 Boeve BF, Molano JR, Ferman TJ, et al. Validation of the Mayo sleep questionnaire to screen for REM sleep behavior disorder in an aging and dementia cohort. Sleep Med 2011;12:445-53.

29 Johns MW. A new method for measuring daytime sleepiness: the Epworth Sleepiness scale. Sleep 1991;14:540-5

30 Thorgrimsen L, Selwood A, Spector A, et al. Whose quality of life is it anyway? the validity and reliability of the quality of Life-Alzheimer's disease (QoL-AD) scale. Alzheimer Dis Assoc Disord 2003;17:201-8.

31 Boström F, Jönsson L, Minthon L, et al. Patients with dementia with Lewy bodies have more impaired quality of life than patients with Alzheimer disease. Alzheimer Dis Assoc Disord 2007;21:150-4.

32 Williams A, Sera L, McPherson ML. Anticholinergic burden in hospice patients with dementia. Am J Hosp Palliat Care 2019;36:222-7.

33 Kolanowski A, Fick DM, Campbell J, et al. A preliminary study of anticholinergic burden and relationship to a quality of life indicator, engagement in activities, in nursing home residents with dementia. $J$ Am Med Dir Assoc 2009;10:252-7.

34 O'Mahony D, O'Sullivan D, Byrne S, et al. STOPP/START criteria for potentially inappropriate prescribing in older people: version 2. Age Ageing 2015;44:213-8.
35 Dow J, Robinson J, Robalino S, et al. How best to assess quality of life in informal carers of people with dementia; a systematic review of existing outcome measures. PLoS One 2018;13:e0193398.

36 Holland JM, Currier JM, Gallagher-Thompson D. Outcomes from the resources for enhancing Alzheimer's caregiver health (reach) program for bereaved caregivers. Psychol Aging 2009;24:190-202.

37 Steffen AM, McKibbin C, Zeiss AM, et al. The revised scale for caregiving self-efficacy: reliability and validity studies. J Gerontol B Psychol Sci Soc Sci 2002;57:P74-86.

38 Steffen AM, Gallagher-Thompson D, Arenella KM, et al. Validating the revised scale for caregiving self-efficacy: a cross-national review. Gerontologist 2019;59:e325-42.

39 Zimet GD, Powell SS, Farley GK, et al. Psychometric characteristics of the multidimensional scale of perceived social support. J Pers Assess 1990:55:610-7.

40 Hardan-Khalil K, Mayo AM. Psychometric properties of the multidimensional scale of perceived social support. Clin Nurse Spec 2015;29:258-61.

41 Hughes S, Lepore M, Walberg D. NADRC: evaluating dementia services and supports: instrument resource list. administration for community living, U.S. department of health and human services, 2018. Available: https://nadrc.acl.gov/node/70

42 Carver CS. You want to measure coping but your protocol' too long: Consider the brief cope. Int J Behav Med 1997;4:92-100.

43 Moore KJ, Davis S, Gola A, et al. Experiences of end of life amongst family carers of people with advanced dementia: longitudinal cohort study with mixed methods. BMC Geriatr 2017;17:135.

44 Bédard M, Molloy DW, Squire L, et al. The Zarit burden interview: a new short version and screening version. Gerontologist 2001;41:652-7.

45 Leggett AN, Zarit S, Taylor A, et al. Stress and burden among caregivers of patients with Lewy body dementia. Gerontologist 2011;51:76-85

46 Marwit SJ, Meuser TM. Development of a short form inventory to assess grief in caregivers of dementia patients. Death Stud 2005;29:191-205.

47 Rosa RDLda, Simões-Neto JP, Santos RL, et al. Caregivers' resilience in mild and moderate Alzheimer's disease. Aging Ment Health 2020;24:250-8.

48 Pessotti CFC, Fonseca LC, Tedrus GMdeAS, Tedrus G, et al. Family caregivers of elderly with dementia relationship between religiosity, resilience, quality of life and burden. Dement Neuropsychol 2018;12:408-14.

49 Teno JM, Freedman VA, Kasper JD, et al. Is care for the dying improving in the United States? J Palliat Med 2015;18:662-6.

50 Gallagher R, Krawczyk M. Family members' perceptions of endof-life care across diverse locations of care. BMC Palliat Care 2013;12:25.

51 Harris PA, Taylor R, Thielke R, et al. Research electronic data capture (REDCap)--a metadata-driven methodology and workflow process for providing translational research informatics support. J Biomed Inform 2009;42:377-81.

52 Harris PA, Taylor R, Minor BL, et al. The REDCap Consortium: building an international community of software platform partners. $J$ Biomed Inform 2019;95:103208.

$53 \mathrm{Li}$ Z, Frost HR, Tosteson TD, et al. A semiparametric joint model for terminal trend of quality of life and survival in palliative care research Stat Med 2017;36:4692-704.

$54 \mathrm{Li} \mathrm{Z,} \mathrm{Tosteson} \mathrm{TD,} \mathrm{Bakitas} \mathrm{MA.} \mathrm{Joint} \mathrm{modeling} \mathrm{quality} \mathrm{of} \mathrm{life} \mathrm{and}$ survival using a terminal decline model in palliative care studies. Stat Med 2013:32:1394-406.

55 Tibshirani R. Regression shrinkage and selection via the LASSO: a retrospective. J $R$ Statist Soc B 2011;73:273-82.

56 Zhang $\mathrm{C}-\mathrm{H}$. Nearly unbiased variable selection under minimax concave penalty. Ann. Statist. 2010;38:894-942.

57 Barber RF, Candès EJ. A knockoff filter for high-dimensional selective inference. Ann. Statist. 2019;47:2504-37.

58 Gan G, Ma C, Wu J. Algorithms, and applications. ASA-SIAM series on statistics and applied probability. Philadelphia, PA, Alexandria, VA: ASA, SIAM, 2007.

59 Lanza ST, Rhoades BL. Latent class analysis: an alternative perspective on subgroup analysis in prevention and treatment. Prev Sci 2013;14:157-68.

60 Colorafi KJ, Evans B. Qualitative descriptive methods in health science research. HERD 2016;9:16-25. 